\title{
Fonctions morphosyntaxiques et sémantiques de quelques présentatifs en français
}

\author{
Dr. \\ Taha Roushdy
}



é

à

à 
à

《

1)

《

〉)

à

《

1) 


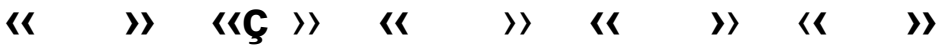

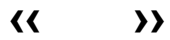

à

à

《 \

$\langle\quad\rangle$

é

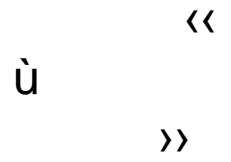

$\langle>\rangle$

$>$ 邹

《 $\quad 1$ 
anaphorique dans la phrase française. De plus, lorsque cette forme démonstrative "ça» anaphorise un énoncé ou une suite d'énoncé, il acquiert de nouvelles propriétés. Le remplacement de "ça» par «cela», ici, est syntaxiquement acceptable et la phrase reste grammaticalement correcte et plus soutenue : «Vous voulez qu'on lui laisse cela dans l'estomac? ")

\section{Ça dans l'interrogation elliptique}

La phrase elliptique, en général, est une forme essentielle dans le discours français. Les auteurs du Dictionnaire de linguistique et des sciences du langage (174) définissent cette catégorie en disant « On qualifie d'elliptiques certaines phrases incomplètes, inachevées dans lesquelles il manque un élément structurel.) Si nous observons cette phrase: Ali est généreux comme son père, nous allons découvrir qu'il y a une partie élidée, sans qu'il y ait aucun changement sémantique de la structure phrastique. La partie supprimée est: "son père est généreux.» qu'on l'appelle (le comparé), tandis que Ali est (le comparant). Le présentatif ça joue le même rôle dans la phrase interrogative. II remplace, dans la plupart des cas, une phrase elliptique s'il est précédé d'un mot interrogatif: Qui ça?- Quand ça?- Où ça?, etc.

-Quand est-ce qu'il est absent? interrogative complète

-Quand ça?

-Forme elliptique

-Quatre soldats ont été tués hier soir. ----forme déclarative

-Où ça? Forme elliptique 
Ça implique beaucoup d'interprétations anaphoriques dans les deux exemples précédents. II peut remplacer tous les éléments de la phrase interrogative sauf le mot interrogatif lui-même, qu'il soit adverbe ou pronom. Cette forme est toujours utilisée dans la conversation de la langue courante ou le dialogue dans l'œuvre romanesque. Syntaxiquement, Les phrases elliptiques sont incomplètes, mais du point de vue sémantique elles ont le même sens que les formes complètes, parce que l'anaphore assure sa reprise sémantique déjà exprimée. On peut faire l'ellipse en utilisant "ça» avec un adverbe interrogatif dans les phrases suivantes:

$-<<$ Pourquoi avez-vous fait ça, madame? Oh! Pourquoi avez-vous fait ça? (La P... respectueuse)

Nous pouvons abréger ces deux questions en deux mots sans aucun changement sémantique.

-Pourquoi ça, madame? Oh! Pourquoi ça?

L'interrogation simple est une catégorie grammaticale qui se contente d'un seul mot interrogatif comme Où?Qui?- Comment?- Quand?, etc. Mais on peut renforcer cette forme par le présentatif «ça» pour éclaircir l'opération de la parole et rendre les énoncés elliptiques. Cela signifie que les mêmes propriétés syntaxiques s'appliquent aux deux types d'interrogation: Où ça?- Qui ça?- Comment ça?Quand ça? La phrase interrogative peut passer par trois changements comme dans l'exemple suivant:

-J'habite Tanta.

-Vous habitez où? ----------------Tanta

-Où? ------------------------------Tanta

-Où ça? ---------------------------Tanta 
Les deux questions: Où ça?, O ?, peuvent donc être les questions d'une réponse déjà connue pour demander plusieurs informations. Voici un exemple qui éclaircit cette idée:

-Nous allons rencontrer Ahmed et Mona.

Le destinataire qui ne connait pas les deux personnes peut répondre:

\section{Qui ça?}

Dans l'interrogation indirecte, le présentatif «ça» n'a pas les mêmes fonctions syntaxiques d'une interrogation simple; il est possible d'insérer les adverbes interrogatifs si- où- pourquoi dans le discours indirect, mais il est complètement interdit d'ajouter «ça» avec le mot interrogatif. Voici un exemple:

-<<Maman, j'étais partie presque tout de suite après l'avoir embrassée et c'est pourquoi il me semblait que c'était encore sa personne qui gisait, solitaire, dans le froid d'une morgue.>> (

, p.139)

Si nous ajoutons «ça» après le mot interrogatif pourquoi *(c'est pourquoi ça il me semblait...), la phrase devient agrammaticale et sa sémanticité porte une autre interprétation. Nous ne pouvons pas dire également (Je me demande où ça vous habitez.)Mais les français font souvent des erreurs en parlant, j'ai entendu à Grenoble quelqu'un qui proférait une phrase méritant l'explication. II a dit: «Je me demande où ça se trouve.») Quand il cherchait l'adresse du magasin (Leader Price).

Le présentatif «ça» peut être donc elliptique dans la plupart des formes interrogatives simples, sans 
supprimer la structure sémantique et syntaxique de la phrase française. Dans ce cas, sa présence est perçue comme un support langagier parce qu'il donne à la phrase une valeur directive et orientative, c'est-à-dire qu'il oriente le destinataire vers le sens correct.

En fait, ça comporte beaucoup de fonctions dans le texte. Il peut jouer le rôle des pronoms possessifs. Voici un exemple:

- Il me fallait une voiture plus grande que la tienne.

-ll me fallait une voiture plus grande que ça.

Le même pour les pronoms démonstratifs:

-Il me fallait une voiture plus grande que celle-ci.

-ll me fallait une voiture plus grande que ça.

Le rôle de «ça» à la fin de la phrase est pertinent pour éviter la répétition ou remplacer un syntagme nominal. II est également anaphorique, parce qu'il remplace le SN déjà cité (voiture). Si nous supprimons "ça»» de n'importe quelle phrase mentionnée ci-dessus, la construction anaphorique devient zéro et l'ambiguïté d'interprétation sera augmentée et susceptible de perturber le destinataire.

Le rôle de «ça») n'est pas souvent convenable dans quelques phrases. Voici un exemple:

-J'ai l'impression que mon épouse est plus bavarde que cette femme.

Le présentatif «ça» n'est pas anaphorique dans cet énoncé et il ne peut pas remplacer cette femme. Le sens de la phrase reste ambigu et exige beaucoup 
d'éclaircissements si nous commutons cette femme par «(ça»).

*-J'ai l'impression que mon épouse est plus bavarde que ça.

Malgré l'interprétation comparative de cette phrase, le lecteur attentif demande toujours l'identification de comparé pour savoir la technique énonciative de la parole.

Nous pouvons dire que le présentatif «ça» est également une forme multifonctionnelle parce qu'elle jouit d'une malléabilité catégorielle bien attestée dans le discours français. II se trouve presque dans la plupart des catégories grammaticales utilisées dans langue parlée.

Enfin, la qualité discursive de «ça» lui donne une présence massive dans la langue orale ainsi que dans les dialogues romanesques. Son rôle dans la conversation fait de lui le présentatif en usage de la langue française parlée. N'oublions pas que le présentatif «cela» remplace obligatoirement «ça» dans la narration littéraire.

\section{Ceci et cela vs ça}

Les deux présentatifs «ceci» et «cela» n'ont pas la même priorité que c'est et ça, mais ils tiennent un rôle important dans le texte français. Pour La Grammaire expliquée du français (1994- 58):«Les pronoms ceci et cela appartiennent à la langue écrite et à une langue orale soutenueı. Ils s'opposent toujours dans leur signification et dans leur emploi. Nous avons constaté en regardant les différents débats sur TV5, que malgré leur rapprochement syntaxique, les deux présentatifs 
ceci et cela ne sont pas interchangeables dans la conversation. Ceci est un pronom démonstratif renforcé par l'adverbe «(ci») qui désigne ce qui est plus proche de celui qui parle. II peut aussi montrer une déclaration, une explication, une énumération, etc. II indique que l'action va se passer sur-le-champ. Voici quelques exemples:

-Ouvre ta bouche et avale ceci!

-Expliquez bien ceci aux élèves: ils sont faibles et ne sont pas capables de composer une simple petite phrase.

-Ceci n'est pas important.

Les trois cas de «ceci» révèlent que les choses concernées, qu'elles soient concrètes ou abstraites, se trouvent dans le moment de l'énonciation du locuteur.

Cela, au contraire, indique ce qui est éloigné de la personne qui tient la responsabilité de la parole. II sert également ce qui précède, ce qui vient d'être écrit ou dit. II est l'équivalent de «ça» dans presque tous ses cas syntaxiques. Selon Grevisse (2004:1024) «Les deux formes sont, du point de vue syntaxique, presque toujours interchangeables, mais «cela» dans la langue écrite et «ça» dans la langue parlée.» II existe dans le code oral comme dans le code écrit. Ce pronom peut également remplacer le présentatif «ça» comme dans les deux exemples suivants:

-Donnez-moi ça!

-Donnez-moi cela!

-Nous lui avons promis ça!

-Nous lui avons promis cela! 
Par ailleurs, l'emploi de «cela» dans les deux exemples précédents révèle les différents genres discursifs et la reformulation introduite par les deux phénomènes nous mène à la compréhension en facilitant ainsi la communication. Dans La P... respectueuse de Sartre, le présentatif «cela» remplace facilement «ça» dans presque toutes les phrases. Voici un exemple:

-Lizzie: Qu'est-ce que ça veut dire? ----Qu'est-ce que cela veut dire?

-Le nègre: ça veut dire qu'il ne me reste plus qu'à courir en rond jusqu'à ce qu'ils m'attrapent.

Le nègre peut dire: «cela veut dire....) et le sémantisme est le même, ainsi que la structure syntaxique. Mais «(ceci») est loin de tout remplacement. Donc les deux formes sont complètement différentes. Dubois dans (Grammaire structurale du français: nom et pronom: 1965, 167) a dit que « cela et ceci n'ont pas la même fréquence». Ceci et ça sont également différents en situation de la parole et en référence. Contrairement à ceci, cela peut être remplacé par «ça» dans la langue parlée surtout quand on répète «ça» plusieurs fois dans un seul contexte. Dubois fait remarquer que « [Cela] apparait dans la langue parlée chaque fois que l'on fait répéter une phrase qui contenait [sa], ou lorsque le locuteur allonge l'énoncé ou le marque d'une intonation à valeur stylistique.») Voici deux exemples:

$-<<$ Elle a eu un bref sanglot:« La seule chose qui me console», m'a-t-elle dit, " c'est que moi aussi je passerai par là. Sans ça (cela), ça (cela) serait trop injuste!» (Une mort très douce p.143) 
-<<C'est donc pour ça (cela) que tes yeux brillaient. Ça (cela) t'excitait, hein? Avec un père qui est si bon. >>

Dans la plupart des cas le remplacement est acceptable mais dans les conversations qui appartiennent à registre familier et populaire, la commutation entre les deux catégories sera difficile. Cela ne peut pas remplacer ça dans les variations suivantes:

-Ça va bien? ${ }^{\star}$ Cela va bien?

-Comment allez-vous?

-Comme ci, comme ça. -------- Comme ci, comme cela.

- Est-ce que ça travaille comme vous voulez? ------`Estce que cela travaille comme vous voulez?

-Ça roule ${ }^{*}$ Cela roule!

Nous avons constaté que les commutations sont plus ou moins possibles dans les énoncés précédents.

Ça possède également une autre valeur dans la conversation. II peut facilement métamorphoser le groupe nominal animé en groupe nominal inanimé comme dans les exemples suivants:

Ça va très bien. / II va très bien. Ou Celui-ci va très bien.

Ça n'arrête pas. / II n'arrête pas. Ou Celui-ci n'arrête pas.

Ça--------------Syntagme nominal inanimé

II et Celui-ci---------------Syntagme nominal animé

Dubois (1965 168) commente cette commutation en disant:<<ça est une forme marquée de IL et une forme 
inversement marquée de celui-ci; le terme est alors disponible pour une valeur affective. II existe un jeu entre les deux systèmes de référence, qui sont sousensembles de l'ensemble démonstratif. >>

Enfin, après l'analyse de bon nombre de fonctions de ça. II s'avère que ce présentatif a d'autres applications que nous avons passées sous silence afin que les autres chercheurs les traitent plus tard. II sera question dans l'étude suivante d'aborder les trois présentatifs «voici», «voilà» et «il y a» pour éclaircir d'une manière succincte leurs rôles principaux dans la langue française.

\section{Voici et voilà: proximité/éloignement}

Voici et voilà ont un caractère démonstratif clairement assuré et sont considérés selon Alain Rabatel (p.63): des «présentatifs purs». Du point de vue grammatical, les deux présentatifs sont invariables. La plupart des livres de grammaire et les grands ouvrages de linguistique affirment clairement que les deux présentatifs voici et voilà possèdent la même origine. Littéralement, les deux formes signifient "vois ici»» pour voici et «vois là» pour voilà. Mais malgré leur rapprochement syntaxique, leurs emplois sont entièrement différents l'un de l'autre. Voilà est une forme plus courante et plus connue. C'est est un élément commun entre le texte narratif et le dialogue. Parfois les locuteurs utilisent «voilà» au lieu de «voici», pour faciliter la parole. Mais les deux présentatifs ont un rapport étroit avec le syntagme nominal qui les suit dans la parole. Ils font partie intégrante de la phrase. Pour l'emploi de «voici», nous avons quelques exemples: 
-Voici ma maison.

-Voici mon livre de géographie.

Comme le verbe, ces deux présentatifs acceptent les compléments, mais les plus courants sont:

-Voilà mon portable---------Le voilà.

-Voici Hala----------------La voici.

-Voici une pomme----------En voici une.

-Voici des fruits En voici.

$-<<$ Elle est dans la salle de bains. (Elle sort. Fred ouvre rapidement les tiroirs de la table et fouille, Lizzie rentre avec la cravate.) La voilà! Attends. > (La p... respectueuse, p.22)

Les compléments qui suivent ces catégories sont une partie essentielle de la construction présentative. Ils condensent la parole en gardant son sémantisme. Ces compléments, comme on a déjà dit, sont des SN. Les propositions relatives sont considérées comme un support syntaxique de voici et voilà dans le texte français. Voici quelques exemples:

-Voilà ce que vous m'avez dit.

-Voici l'homme dont j'ai besoin.

$-<<$ Je ne sais pas, moi: sa cruche. Elle devait avoir une cruche. Je voudrais une vieille grand-mère pour lui faire pendant. Elle tricoterait ou elle raconterait une histoire à ses petits-enfants. Ah! Je vais tirer les rideaux et ouvrir la fenêtre. (Elle le fait.) Ce qu'il fait beau! Voilà une journée qui commence. (Elle s'étire.)>> ( , p.20) 
Voici est un présentatif qui accepte l'infinitif, mais «voilà» est exclu de cette construction grammaticale :

-*Voilà boire le reste de l'eau.

-Voici venir la pluie.

On ne peut pas dire:

-*Voilà venir la pluie.

L'énoncé qui contient «voici» est vu dans son déroulement au moment de l'énonciation ou anticipe les événements pour les moments à venir. Dans l'exemple ci-dessus l'infinitif venir qui suit le présentatif «voici» montre le procès en cours. Cet emploi a été bien souligné par les grammairiens, dont Togeby, $\mathrm{K}$. (1983:55) qui affirme que: « la proposition infinitive ne se trouve qu'après voici, et ne contient que le verbe venir: "Voici venir le mensonge»».

Nous pouvons dire que les deux phénomènes présentatifs sont liés indissociablement à l'énonciateur qui est toujours présent dans la construction nominale de la phrase. La plupart des cas de «voici»» et «voilà»» apparaît dans le dialogue pour montrer quelque chose ou pour affirmer une idée absente. Voilà un autre exemple dans une conversation où le présentatif «voilà» traduit un sentiment de satisfaction ou un acte d'acquiescement à l'égard d'une réponse.

<<Mme Maure: Mais le château date du dix-septième siècle. Comment ça se fait?

Le Guide: C'est très simple. Elle vient de l'ancien château familial, qui n'existe plus, mais qui a été construit au quatorzième siècle. Le comte Henri de 
Beaufort, qui a fait construire ce château-ci, a gardé certains meubles de l'autre.

M. Maure: Ah, voilà.

Mme Maure: Merci du renseignement, Mademoiselle.

Le Guide: A votre service, Messieurs-Dames. >> ( , p.102)

Le présentatif (Ah, voilà) qui apparaît à la fin de ce dialogue «voilà conclusif»" ${ }^{1}$ contient beaucoup de valeurs: la satisfaction de l'interlocuteur quant à l'explication énoncée par le locuteur, la découverte de nouvelles informations et de résumer de vouloir dire de l'interrogateur et la clôture d'une interaction verbale entre plusieurs personnes. Ce phénomène camoufle des réactions de $\mathrm{M}$. Maure et met en valeur la situation.

On ne peut pas remplacer le présentatif «voilà» par "voici»» dans ce dialogue parce que "voilà» donne un sens collectif à l'énoncé et harmonise le fil conducteur des phrases. Dans le dialogue, susmentionné il y a des informations abstraites alors que voilà est le seul présentatif le plus adéquat. Le présentatif «voici» rapproche toujours les distances entre le locuteur et l'interlocuteur et désigne quelque chose de présent. Voici un exemple:

-Bonjour Monsieur, voici le loyer pour le mois prochain.

Le locataire tend sa main en donnant le loyer à son propriétaire et le présentatif «voici» renforce la phrase par son caractère de simultanéité. Mais le présentatif «voilà» peut à lui seul former un énoncé qui constitue

-Terme emprunté de Riegel et autres: Grammaire méthodique du français, p.454 
une réponse à une telle question. L'exemple suivant éclaircit cette idée:

-Où est le voleur?

\section{-Voilà! Voilà!}

-Où est votre carte d'identité?

\section{-Voilà!}

Voilà apparaît beaucoup dans les phrases à modalité exclamative pour renforcer positivement les énoncés. Dans les deux positions ci-dessus «voilà» a un rapport très étroit avec les moments de l'énonciation.

Les deux formes présentatives sont quasiment absentes dans la narration passée surtout dans les récits littéraires. Mais pour guider le lecteur à comprendre l'histoire, ils paraissent rarement dans quelques passages narratifs chez les grands écrivains comme Zola, Flaubert, Balzac, etc. voici un exemple:

$-<<$ ll cherchait, ne trouvait pas. Mais les plus fameux chirurgiens se trompaient bien. Voilà ce qu'on ne voudrait jamais croire! On allait rire, au contraire, clabauder! Cela se répandrait jusqu'à Forges!>> (Madame Bovary, p.252)

\section{La diversité polyvalente du présentatif il y a}

II y a est l'un des présentatifs qui mérite d'être étudié afin qu'on sache son rôle sémantique et syntaxique dans la phrase française. II accepte tous les changements temporels surtout avec les différentes formes verbales. Ce présentatif possède beaucoup de fonctions et son emploi est célèbre dans tous les milieux sociaux, ainsi que dans le domaine littéraire. Ce phénomène est un vrai nœud verbal parce qu'il contient 
le verbe avoir dans sa composition. II est également une forme lexicalisée parce qu'il est très proche d'une préposition. Voici deux exemples:

$-<<$ lls ont mis des sentinelles aux deux bouts de la rue et ils fouillent toutes les maisons. Tu avais bien besoin de venir ici. Il y a sûrement quelqu'un qui t'a vu entrer dans la rue. $>$ (La p... respectueuse, p.59)

-L'avion a décollé il y a deux heures.

Malgré sa structure verbale, le présentatif «il y a» ne varie pas en personne. II fait partie des verbes impersonnels: «il faut», «il existe», «il reste», etc. Mais il accepte les pronoms personnels comme le pronom 《en» qui prend sa position avant le verbe avoir. Nous avons deux exemples:

-<<Des amies ont téléphoné, j'ai répondu. «Eh bien! Lui ai-je dit. Ça n'arrête pas. La reine d'Angleterre ne serait pas plus gâtée: des fleurs, des lettres, des bonbons, des coups de téléphone! II y en a des gens qui pensent à toilı (Une mort très douce, p.34)

$-<<$ Pas du tout. Les deux nègres se tenaient tranquilles et parlaient entre eux; ils ne m'ont même pas regardée. Après, quatre blancs sont montés et il y en a deux qui m'ont serrée de près. > (La p... respectueuse, p.35)

Comme le présentatif «c'est», «il y a» est toujours accompagné par un syntagme nominal. Voici deux exemples:

-<<ll y a quatre pensionnaires, vêtues de noir, et deux externes en toilette blanche: maman et une de ses amies. >> (Une mort très douce, p.46) 
-<<Bien sûr: je les attire, il y a des natures comme ça. > (La p... respectueuse, p.31)

Il apparaît souvent dans la conversation en posant une question pour demander beaucoup d'éclaircissements et savoir quelque renseignement nouveau. Voici un exemple qui prouve cette idée.

$-<<$ Qui est-ce qui te cause de ça? Je te demande si tu es content, tu peux bien me répondre gentiment. Qu'est-ce qu'il y a? Tu n'es pas vraiment content? Oh! Ça m'étonnerait, tu sais, ça m'étonnerait. (La p... respectueuse, p.23)

La question: (Qu'est-ce qu'il y a?) comporte le présentatif «il y a» qui désigne plusieurs cas: soit on s'interroge sur des personnes, soit sur une situation. II équivaut: De quoi s'agit-il?

Il est utilisé dans la phrase relative pour confirmer la justification de l'information. Voici un exemple:

$-<<$ lls ont mis des sentinelles aux deux bouts de la rue et ils fouillent toutes les maisons. Tu avais bien besoin de venir ici. Il y a sûrement quelqu'un qui t'a vu entrer dans la rue. Voilà. C'est à nous. Ils montent. >> (La p... respectueuse, p.69)

Le présentatif «il y a» apparaît dans la phrase complétive pour donner une réponse et éclaircir un nouvel état. L'exemple suivant justifie cette idée:

$-<<$ Ah! Maintenant, je me sens bien. Vraiment bien. II y a longtemps que je me sens bien. >> (Une mort très douce, p.123)

Nous avons constaté une différence claire entre l'emploi de «il y a » et les deux présentatifs 
homologues «voici» et «voilà)» qui acceptent des syntagmes nominaux comportant n'importe quel article et pratiquement n'importe quel déterminant. Voici quelques exemples:

-Voici mes frères.

-Voilà les deux oiseaux.

-Voici mon directeur.

Mais le présentatif «il y a» peut prendre un article indéfini ou un déterminant indiquant une quantité. Nous avons beaucoup d'échantillons:

-Il y a un rat qui passe par ici.

-II y a des paysans dans les champs.

-Il y a dix élèves dans la classe.

Dans son usage non verbal, le présentatif «il y a» est une expression lexicalisée qui fonctionne exactement comme une préposition de temps. Selon Riegel Martin (1994:456) le présentatif il y a sert à «introduire des compléments circonstanciels de temps; il joue alors le rôle d'une préposition>> Voici deux exemples:

-Mon père est mort il y a cinq ans.

-François Mitterrand est décédé il y a presque vingtcing ans.

Comme n'importe quel complément circonstanciel de temps, le présentatif polyfonctionnel «il y a» est précédé quelquefois de la préposition «de» qui donne une information historique ou reflète les vestiges du monde d'autrefois. Voici un exemple:

-Des monuments égyptiens d'il y a deux mille ans. 
-Des statuettes pharaoniques d'il y a trois mille ans.

La négation est l'ennemi permanent de cette forme présentative. On peut dire: Elle est descendue il y a quatre heures. Mais on ne peut pas dire: Elle n'est pas descendue il y a quatre heures. La négation ici arrête la fonction principale de il y a. Mais on peut dire facilement: Elle n'est pas descendue il y a quatre heures, mais six heures. II est à noter que «voici») et «voilà» ne remplacent pas «il y a») surtout dans la langue parlée, sauf dans quelques cas exceptionnels. On constate cette opposition dans les mêmes exemples précédents:

*-Elle est descendue voici quatre heures.

*-Elle n'est pas descendue voilà quatre heures.

Les deux dernières formes sont agrammaticales et leur sens dérègle le comportement normal d'un système. Mais on peut dire facilement: Voici cinq ans que mon père est mort. Les présentatifs polyfonctionnels il y a et son homologue c'est fortifient la phrase française en faisant une liaison logique entre ses différents éléments. Jean- Michel Adam (1990-168) explique cette idée en disant: «ll y a et c'est sont deux organisateurs qui, dans le cadre d'une énumération, fonctionnent de la même manière; tous deux soulignent l'enchaînement et l'empaquetage des propositions..)

\section{Conclusion}

Au terme de ce travail, nous pouvons affirmer que les présentatifs que nous venons d'étudier remplissent la tâche de la présentation des noms, objets, actions ou idées. Ces phénomènes renferment la quasi-totalité des énoncés français, jadis comme actuellement, aussi 
bien à l'oral qu'à l'écrit et ne cessent de se réactualiser en fonction de l'usage toujours renouvelable de la langue.

Ce travail pourrait être complété et enrichi par d'autres études plus approfondies.

\section{Bibliographie sélective}

Arrivé Michel et les autres,

Flammarion, Paris, 1986.

Cadiot, P., «De quoi ça parle? A propos de la référence de ça, pronom-sujet», Le Français moderne, 3/4: 174192. 1988.

Charaudeau, $\mathrm{P}$.

Hachette, 1992.

Chevalier Jean-Claude,

no 1, 1969.

, In Langue française,

Dubois Jean et les autres,

$$
\text { , Larousse, } 1994 .
$$

Dubois Jean, : nom et pronom, Larousse, 1965.

Jean-Michel Adam, Mardaga, 1990.

Gardes-Tamine, J., Armand Colin, 1998.

Godart-wendling, B., ? Revue de sémantique et pragmatique, Numéro 7, 2000.

Grevisse, M., Le bon usage, Duclot, 2004. 
Kleiber, G., In Langage,

Numéro 97, 1990.

Porquier, R., , Le français dans le monde, Numéro 91, 1972.

Rabatel M. Alain,

In Langue française, no 128, 2000.

Rabatel M. Alain,

d'Orleans, 2000.

, presses de l'Université

Riegel, M. et les autres, , PUF, 1994.

Togeby, K., Volume III: les constructions impersonnelles du verbe et la construction des verbes, Copenhague, 1983. 
7rIT

رقه الإيداع 
7rIT

رقه الإيداع 
7rIT

رقه الإيداع 
7rIT

رقه الإيداع 
7rIT

رقه الإيداع 
7rIT

رقه الإيداع 
7rIT

رقه الإيداع 
7rIT

رقه الإيداع 
7rIT

رقه الإيداع 
7rIT

رقه الإيداع 
7rIT

رقه الإيداع 
7rIT

رقه الإيداع 
7rIT

رقه الإيداع 
7rIT

رقه الإيداع 